\title{
BMJ Open Drug-induced sleep endoscopy compared with systematic adenotonsillectomy in the management of obstructive sleep apnoea in children: a systematic review and meta- analysis protocol
}

\author{
Anne-Sophie Prévost, ${ }^{1}$ Mathieu Hylands, ${ }^{2}$ Mireille Gervais, ${ }^{1}$ Jean-Paul Praud, ${ }^{3}$ \\ Marie-Claude Battista, ${ }^{4}$ Stéphanie Déziel-Malouin, ${ }^{5}$ Monia Lachance, ${ }^{5}$ \\ Francois Lamontagne ${ }^{6}$
}

To cite: Prévost A-S, Hylands M, Gervais $\mathrm{M}$, et al. Druginduced sleep endoscopy compared with systematic adenotonsillectomy in the management of obstructive sleep apnoea in children: a systematic review and metaanalysis protocol. BMJ Open 2019;9:e028242. doi:10.1136/ bmjopen-2018-028242

- Prepublication history and additional material for this paper are available online. To view these files, please visit the journal online (http://dx.doi. org/10.1136/bmjopen-2018028242).

Received 28 November 2018 Accepted 16 August 2019

D) Check for updates

(c) Author(s) (or their employer(s)) 2019. Re-use permitted under CC BY-NC. No commercial re-use. See rights and permissions. Published by BMJ.

For numbered affiliations see end of article.

Correspondence to Dr Anne-Sophie Prévost; anne-sophie.prevost@ usherbrooke.ca

\section{ABSTRACT}

Introduction Obstructive sleep apnoea affects up to $6 \%$ of children worldwide. Although current guidelines recommend systematic tonsillectomy and adenoidectomy, many children do not benefit from these interventions. Drug-induced sleep endoscopy (DISE) allows the dynamic evaluation of patients' airways to identify the specific anatomic sites of obstruction. This intervention can potentially guide subsequent invasive procedures to optimise outcomes and minimise the number of children exposed to unnecessary operations.

Methods and analysis We will identify randomised controlled trials and controlled observational studies comparing DISE-directed interventions to systematic tonsillectomy and adenoidectomy in paediatric populations. We will search MEDLINE, EMBASE, CINAHL, CENTRAL as well as clinical trial registries and conference proceedings (initial electronic search date 9 October 2018). Screening, data extraction and risk of bias assessments will be performed in duplicate by independent reviewers. We will use the Grading of Recommendations Assessment, Development and Evaluation approach to assess the overall quality of evidence and present our results. Ethics and dissemination Ethics approval is not required for this systematic review of published data. This review will be presented according to the Preferred Reporting Items for Systematic Reviews and MetaAnalyses guidelines. We will present our findings at otorhinolaryngology conferences and publish a report in a peer-reviewed journal.

PROSPERO registration number CRD42018085370.

\section{INTRODUCTION}

Obstructive sleep apnoea (OSA) affects 1\%-6\% of school-aged children. ${ }^{12}$ Left untreated, this condition is associated with neurocognitive and behavioural disorders, cardiovascular consequences, failure to thrive and poor quality of life. $^{2-6}$

\section{Strengths and limitations of this study}

- This review is prospectively registered, includes a detailed search strategy and explicit, prespecified inclusion and exclusion criteria.

- We will provide transparent and clear reporting of our findings using the Grading of Recommendations Assessment, Development and Evaluation approach.

- This systematic review will offer a rigorous, comprehensive assessment of the literature pertaining to the use of drug-induced sleep endoscopy for a common paediatric condition.

- One of our main outcome measures, the apnoea-hypopnoea index, is an imperfect metric which does not consider variables such as cardiovascular complications and daytime functioning. It is nonetheless rigorously standardised and commonly used.

- The number and methodological quality of available studies will likely limit our conclusions.

Nocturnal laboratory polysomnography (PSG) is the diagnostic gold standard for OSA in children with either an obstructive apnoea index (OAI) $>1$ /hour or an obstructive apnoea-hypopnoea index (oAHI) of $>1.5$ / hour. $^{27-9}$ The AHI is an imperfect metric, as it does not consider relevant variables such as cardiovascular complications or daytime functioning. It is nonetheless the outcome most likely to be consistently reported, given the standardisation of scoring rules by the American Academy of Sleep Medicine. ${ }^{10}$ Tonsillectomy and adenoidectomy is recommended by the American Academy of Pediatrics as the first-line therapy for children diagnosed with OSA and adenotonsillar hypertrophy. ${ }^{2}$ However, published data on OSA improvement 
following adenotonsillectomy remain inconclusive, with a variable success rate between $12 \%$ and $83 \% .^{11}{ }^{12}$ Other potential treatment approaches include medications (e.g., nasal corticosteroids, leukotriene antagonists), lifestyle interventions for patients with obesity, continuous positive airway pressure, hypoglossal nerve stimulation, myofunctional therapy, supraglottoplasty, lingual tonsillectomy, nasal surgery, maxillofacial surgery and orthodontic treatment. $^{2}{ }^{13} 14$ Accordingly, tests predicting the response to adenotonsillectomy may help distinguish patients who will benefit from those in whom adenotonsillectomy is more likely to be inefficient and potentially harmful.

Drug-induced sleep endoscopy (DISE) consists of the direct examination of the upper airway using flexible endoscopy under deep sedation to identify specific anatomic sites of obstruction. Drugs that induce a haemodynamically stable near-normal sleep are administered, and sleep and snoring must be maintained by ensuring anaesthetics are within appropriate concentration ranges. DISE can be used as a first-line diagnostic tool to select the best candidates for surgery and reduce the rate of unsuccessful invasive interventions. DISE can also be of benefit to children who fail to improve following adenotonsillectomy. ${ }^{13}$

It is conceivable that DISE may improve rates of OSA cure, if the ensuing surgical intervention is better tailored to a patient's specific anatomic abnormality. Alternatively, it is possible that DISE will only avoid unnecessary adenotonsillectomy in patients who would not have benefited from a surgical intervention either way. In this latter scenario, we would expect similar rates of OSA improvement and decreased surgical morbidity in patients undergoing DISE.

\section{OBJECTIVES}

Our primary objective is to determine whether children with OSA should undergo DISE followed by targeted therapy rather than routine adenotonsillectomy without additional preoperative workup. The latter case reflects the current standard of care. Our primary research question is therefore as follows: In children with OSA, does DISEguided management (surgical and/or non-surgical) lead to improved cure rates (normal PSG), compared with firstline adenotonsillectomy without additional preoperative workup?

Our secondary objective is to determine, within the more limited subgroup of patients that ultimately undergo a surgical procedure, whether those selected with preoperative DISE have improved outcomes. Our secondary research question is therefore as follows: In children with OSA, do surgical interventions guided by preoperative DISE lead to improved cure rates (normal PSG), compared with first-line adenotonsillectomy without further preoperative workup?

\section{METHODS AND ANALYSIS}

\section{Protocol and registration}

The Preferred Reporting Items for Systematic Review and Meta-Analysis Protocols (PRISMA-P) statement guided the design of this protocol (online supplementary appendix A). ${ }^{15}$ PROSPERO registration number: CRD42018085370.

\section{Eligibility criteria}

Participants

Our population is limited to surgically naïve children ( $\geq 1$ and $<18$ years of age) with confirmed OSA or mixed sleep apnoea, defined by an OAI $>1$ /hour or an oAHI $>1.5$ /hour ascertained by PSG. We will exclude studies whose populations include congenital craniofacial malformations, neurological or muscular disease impacting respiratory function (eg, cerebral palsy, muscular dystrophy) and patients with previous airway surgery unless these patients account for $<10 \%$ of the total sample size or there are data available for the subgroup of patients without these characteristics. We will not exclude patients with laryngomalacia.

\section{Intervention/Comparator}

The intervention of interest is DISE performed before a first-line therapy for OSA is attempted. The comparator is adenotonsillectomy for all patients presenting with OSA without preoperative DISE. This procedure removes tissue in the nasopharynx and oropharynx, thereby potentially relieving OSA when these are the sites of obstruction. ${ }^{16}$ Multiple techniques (eg, cold steel, monopolar or bipolar diathermy, coblation) are reported in the literature. ${ }^{17-20}$ Complications of adenotonsillectomy include postoperative bleeding, pain, dehydration, postobstructive pulmonary oedema, velopharyngeal insufficiency and death.

\section{Outcome measures}

The primary outcome will be the normalisation of either the OAI $\leq 1$ /hour or oAHI $\leq 1.5$ /hour. Given that we anticipate that there will be few comparative studies addressing our specific research question, we will not exclude studies based on outcomes assessed. However, we will prespecify which secondary outcomes to include in our formal analysis and Grading of Recommendations Assessment, Development and Evaluation (GRADE) summary tables. Outcomes were selected and prioritised following a consultation with otorhinolaryngologists and patient advocates. We followed GRADE recommendations and favoured patient-important outcomes and those that are not surrogate outcomes. ${ }^{21}$ Outcomes of 'low importance' will not be included in our analysis. Outcomes graded as 'critical' were death and acute postoperative respiratory failure. Outcomes deemed to be 'important but not critical' included the proportion of patients cured of OSA, the proportion of patients undergoing an adenotonsillectomy, postoperative bleeding, the number of interventions requiring general anaesthesia, overall cost and quality of life.

\section{Type of studies}

We will include randomised controlled trials and observational studies that allow comparisons between DISEguided interventions and adenotonsillectomy for all patients (cohort or case-control). Case series and case 
reports will be excluded. We will impose no restriction based on language or publication status.

\section{Search strategy}

We will perform a search in MEDLINE, MEDLINE In-Process, CINAHL, EMBASE and The Cochrane Library (CENTRAL database). The initial electronic search was performed on 9 October 2018 and will be updated as we near the publication of our review. An example of this search strategy is included in the online supplementary appendix B. Other sources that will be searched are ClinicalTrials.gov, reference lists of included studies and conference proceedings from the following major scientific meetings since 1988: American Academy of Otolaryngology-Head and Neck Surgery, International Congress on Pediatric Pulmonology, American Thoracic Society, American Pediatric Societies Meeting and European Respiratory Society Meeting.

\section{Study records}

Two reviewers will independently screen titles and abstracts in duplicate using the Rayyan electronic platform (Qatari Computing Research Institute): https:// rayyan.qcri.org/.

We will proceed to full-text review unless both reviewers agree to exclude a report. Both reviewers will assess fulltext reports independently and in duplicate using the same electronic platform. Disagreements will be resolved by consensus or third-reviewer adjudication.

\section{Data collection}

Both reviewers will use pretested data collection forms to collect data independently and in duplicate. Data of interest include study design, population baseline characteristics, intervention characteristics, clinical outcomes and variables necessary for risk of bias assessment. Disagreements will be resolved by consensus or third-reviewer adjudication.

\section{Risk of bias assessment}

We will use a modified version of the Cochrane Collaboration's tool to assess the risk of bias in randomised controlled trials. ${ }^{22}$ This tool evaluates reports for randomisation, allocation concealment, blinding, loss to follow-up, selective outcome reporting as well as other risks of bias.

For non-randomised trials, we will use the Cochrane Collaboration's ROBINS-I tool. ${ }^{23}{ }^{24}$ This tool is based on the principle that each non-randomised study seeks to reproduce the results of an 'ideal' randomised controlled trial. Sources of bias are defined as the differences between the two studies that significantly alter the results of the non-randomised study. ROBINS-I addresses the following domains as potential sources of bias: confounding, selection bias, intervention classification, deviation from anticipated interventions, missing outcome data, method of measuring outcomes, and selective outcome reporting.

For both randomised and non-randomised studies, if any domain presents a potential source of bias (unclear or high risk of bias), then the report will be graded as high risk of bias. ${ }^{25}$

\section{Summarising data and treatment effect}

We will include comparably homogeneous studies in a random-effects meta-analysis, using the inverse-variance method to assign study weights. ${ }^{26}$ We will use the Review Manager software made available by the Cochrane Collaboration (Review Manager V.5.3).

Dichotomous variables will be calculated using individual study odds ratios and presented as risk ratios with 95\% CIs. Continuous outcomes will be presented as mean differences with associated 95\% CIs. Outcomes reported on different scales, such as quality of life, will be presented according to the previously published recommendations of Thorlund $e t a l^{27}$ These recommendations include the use of two or more complimentary methods to present results in units that are easily interpreted by clinicians, for example, as natural units of a familiar instrument or as a number needed to treat. We will also present these data as standardised mean differences, as a sensitivity analysis. We will analyse and present randomised trials and observational studies separately. There are no conditions under which we will pool results from randomised and non-randomised studies.

If we identify no studies homogeneous enough to be included in a meta-analysis, we will provide a qualitative summary of our findings and justify our rationale.

\section{Prespecified subgroup analyses}

We will evaluate study heterogeneity qualitatively by assessing whether study populations, interventions and settings are comparable across studies. The following characteristics will be considered: baseline AHI, Brodsky score, body mass index (BMI), age, sex, ethnicity and socioeconomic status. These categories will constitute the basis for subgroup analyses.

We will evaluate heterogeneity quantitatively using a $\chi^{2}$ test for homogeneity as well as Higgins' and Thomson's $\mathrm{I}^{2}$ statistic. Regardless the degree of heterogeneity identified, we will perform the following limited subgroup analyses:

1. Baseline OSA severity: mild $(1.5<\mathrm{AHI}<5$ or $1 \leq \mathrm{OAI} \leq 5)$, moderate $(5 \leq \mathrm{AHI}$ or $\mathrm{OAI}<10)$ and severe (AHI or OAI $\geq 10$ /hour), hypothesising that more severe OSA will be more likely to benefit from DISE-directed therapy.

If the number of eligible studies is sufficient, we will also explore heterogeneity using quantile regression to analyse apnoea severity as a continuous variable. We will require at least 10 studies in the meta-analysis in order to perform a quantile regression, as recommended by Cochrane guidelines. ${ }^{22}$ Moreover, studies included in this analysis will need to report the estimated treatment effect, associated variance and covariate values. ${ }^{28}$ In order to account for the residual heterogeneity between studies, we will perform a random-effects meta-regression. 
2. Patients' Brodsky score from 0 to 4 , hypothesising that patients with a lower Brodsky score will be more likely to benefit from DISE-directed interventions.

3. Obese ( $\geq 95$ th percentile for BMI) versus patients without obesity, hypothesising that patients with obesity will be less likely to benefit from DISE-directed interventions.

4. Age (1-8years or $>8$ years), hypothesising that older patients will be more likely to benefit from DISE-directed interventions. ${ }^{29}$

5. Ethnicity (white, black or other), hypothesising that African-American patients will be more likely to benefit from DISE-directed interventions.

6. Socioeconomic status (higher vs lower, as defined by individual study authors), hypothesising that patients of lower socioeconomic status will be more likely to benefit from DISE-directed interventions.

We will evaluate the credibility of subgroup effects according to the following: if the subgroup characteristic is present at baseline, whether the comparison is within or between studies, whether the result is statistically significant, whether the result is found consistently across studies and outcomes and whether or not there exists other evidence to support the result. ${ }^{30}$ We will require five or more studies for comparisons between different studies, with each group represented by two or more studies. If the comparison is between subgroups within the same studies, we will require only two studies to perform the analysis.

\section{Sensitivity analyses}

We will conduct a sensitivity analysis excluding studies published as abstracts as well as another excluding studies with 'unclear' or 'high risk of bias'.

\section{Assessment of reporting bias}

If we identify 10 or more eligible studies in a meta-analysis, we will present a funnel plot and either the Egger's test (continuous outcomes) or the arcsine test (dichotomous outcomes) to assess the risk of publication bias, with statistical significance set at $\mathrm{p}<0.05$ for both tests.

\section{Interpretation of results}

The GRADE framework will be used to report the overall quality of evidence and our confidence in estimates of effect. This framework considers the overall risk of bias, imprecision, inconsistency across studies, indirectness and the likelihood of publication bias. ${ }^{31}$ We will classify the quality of evidence for each outcome across studies as being 'very low', 'low', 'moderate' or 'high'.

Confidence in effect estimates will be rated down for overall risk of bias if any study included in the analysis is graded as 'high risk of bias'.

Imprecision refers to the width of the $95 \%$ CI surrounding the overall estimate of effect for an outcome. If clinical decision-making would differ based on whether the upper or lower bound of the CI represented the truth, then the outcome will be rated down for imprecision. ${ }^{33}$
Inconsistency refers to the variation in results across different studies. We will explore inconsistency by assessing the similarity of estimates, overlap of $95 \%$ CIs as well as the $\chi^{2}$ test (with significance established at $\mathrm{p}<0.05$ ) and $\mathrm{I}^{2}$ statistic (with 'substantial heterogeneity' defined as an $\left.\mathrm{I}^{2}>50 \%\right){ }^{34}{ }^{35}$ We will present a transparent rationale justifying the decision to rate down for inconsistency based on these factors and on whether it is explained by our a priori subgroup effects.

Indirectness refers to the degree to which clinical outcomes are surrogate rather than patient-important outcomes. ${ }^{36}$ We will rate down for indirectness if studies fail to address patient-important outcomes directly.

Publication bias refers to the bias that is introduced to a body of evidence if positive studies are more likely to have been published than negative studies. We will rate down for publication bias if the arcsine test, Egger's test or a visual funnel plot are suggestive of significant publication bias. ${ }^{37}$

We will present our results in a 'Summary of Findings' table to represent individual outcomes across studies as well as the quality of evidence for each outcome. ${ }^{38}$ Results from observational studies and randomised trials will be presented separately as different rows within the same table. Our final interpretation of results will rely on the estimate of effect providing the highest degree of certainty (eg, data from high-quality clinical trials if available).

\section{Protocol amendments}

Any amendments to this protocol will be reported with the justification and date of modification.

\section{Patients and public involvement}

Our research question was guided by the lack of consensus on the management of paediatric OSA. We aim to provide guidance on the most effective treatment that minimises adverse effects and risks to patients. Patients were not directly involved in the study design, but a few parents and members of the public were involved in the classification of secondary outcomes.

\section{Ethics and dissemination}

No confidential data will be used, therefore approval by an ethic committee will not be necessary. This systematic review will provide an accurate portrait of the impact of DISE-directed management compared with systematic adenotonsillectomy in the management of paediatric OSA. We will publish our results in a peer-reviewed journal.

The review will be reported according to PRISMA guidelines. $^{15}$

\section{DISCUSSION}

OSA is a common condition that, left untreated, can have profound consequences on future development. The routine adoption of adenotonsillectomy as a first-line treatment aims to mitigate this impact. However, disparities in cure rates between different 
reports suggest that this approach is not optimal for all subgroups of patients. DISE is a promising intervention that may both help select surgical candidates and avoid unnecessary surgeries in children least likely to benefit from adenotonsillectomy.

If we identify high-quality evidence suggesting that preoperative DISE is beneficial, this conclusion will have important implications for practice. In contrast, if we find that the existing evidence is insufficient to provide definitive inferences regarding the effect of DISE before adenotonsillectomy, this review will expose a knowledge gap and provide a strong rationale for further prospective research.

\section{Author affiliations}

${ }^{1}$ Division of Otorhinolaryngology—Head and Neck Surgery, Department of Surgery, Université de Sherbrooke, Sherbrooke, Quebec, Canada

${ }^{2}$ Division of General Surgery, Department of Surgery, Université de Sherbrooke, Sherbrooke, Quebec, Canada

${ }^{3}$ Division of Pediatric Pulmonology—Department of Pediatrics, Université de Sherbrooke, Sherbrooke, Quebec, Canada

${ }^{4}$ Centre de recherche du Centre Hospitalier Universitaire de Sherbrooke, Université de Sherbrooke, Sherbrooke, Quebec, Canada

${ }^{5}$ Department of Anesthesia, Université de Sherbrooke, Sherbrooke, Quebec, Canada ${ }^{6}$ Department of Internal Medicine, Université de Sherbrooke, Sherbrooke, Quebec, Canada

Acknowledgements The search strategy was developed in collaboration with Rachel Couban (McMaster University medical librarian).

Contributors A-SP, MG, FL, M-CB, J-PP and MH conceived the research question. $\mathrm{MH}$ and $\mathrm{FL}$ conceptualised methodological analyses. A-SP and MH wrote the draft protocol and will review articles. All authors reviewed and approved the protocol and will contribute to the writing of the publication.

Funding This project is funded by the Département de chirurgie de l'Université de Sherbrooke.

Competing interests None declared.

Patient consent for publication Not required.

Provenance and peer review Not commissioned; externally peer reviewed.

Open access This is an open access article distributed in accordance with the Creative Commons Attribution Non Commercial (CC BY-NC 4.0) license, which permits others to distribute, remix, adapt, build upon this work non-commercially, and license their derivative works on different terms, provided the original work is properly cited, appropriate credit is given, any changes made indicated, and the use is non-commercial. See: http://creativecommons.org/licenses/by-nc/4.0/.

\section{REFERENCES}

1. Guilleminault $\mathrm{C}$, Lee $\mathrm{JH}$, Chan A. Pediatric obstructive sleep apnea syndrome. Arch Pediatr Adolesc Med 2005;159:775-85.

2. Marcus CL, Brooks LJ, Draper KA, et al. Diagnosis and management of childhood obstructive sleep apnea syndrome. Pediatrics 2012;130:e714-55.

3. Beebe DW, Wells CT, Jeffries J, et al. Neuropsychological effects of pediatric obstructive sleep apnea. $J$ Int Neuropsychol Soc 2004:10:962-75.

4. Lee C-H, Kang K-T, Weng W-C, et al. Quality of life after adenotonsillectomy in children with obstructive sleep apnea: short-term and long-term results. Int $J$ Pediatr Otorhinolaryngol 2015;79:210-5.

5. Amin RS, Kimball TR, Bean JA, et al. Left ventricular hypertrophy and abnormal ventricular geometry in children and adolescents with obstructive sleep apnea. Am J Respir Crit Care Med 2002;165:1395-9.

6. Bonuck K, Parikh S, Bassila M. Growth failure and sleep disordered breathing: a review of the literature. Int J Pediatr Otorhinolaryngol 2006;70:769-78.
7. Marcus $\mathrm{CL}$, Moore $\mathrm{RH}$, Rosen $\mathrm{CL}$, et al. A randomized trial of adenotonsillectomy for childhood sleep apnea. N Engl J Med Overseas Ed 2013;368:2366-76.

8. Uliel S, Tauman R, Greenfeld M, et al. Normal polysomnographic respiratory values in children and adolescents. Chest 2004;125:872-8.

9. Certal V, Camacho M, Winck JC, et al. Unattended sleep studies in pediatric OSA: a systematic review and meta-analysis. Laryngoscope 2015;125:255-62.

10. Berry RB. The AASM manual for the scoring of sleep and associated events: rules, terminology and technical specifications. Am Acad Sleep Med 2018.

11. Costa DJ, Mitchell R. Adenotonsillectomy for obstructive sleep apnea in obese children: a meta-analysis. Otolaryngol Head Neck Surg 2009;140:455-60.

12. Brietzke SE, Gallagher D. The effectiveness of tonsillectomy and adenoidectomy in the treatment of pediatric obstructive sleep apnea/ hypopnea syndrome: a meta-analysis. Otolaryngol Head Neck Surg 2006;134:979-84.

13. Wilcox LJ, Bergeron M, Reghunathan S, et al. An updated review of pediatric drug-induced sleep endoscopy. Laryngoscope Investig Otolaryngol 2017;2:423-31

14. Camacho M, Certal V, Abdullatif $\mathrm{J}$, et al. Myofunctional therapy to treat obstructive sleep apnea: a systematic review and metaanalysis. Sleep 2015;38:669-75.

15. Moher D, Shamseer L, Clarke M, et al. Preferred reporting items for systematic review and meta-analysis protocols (PRISMA-P) 2015 statement. Syst Rev 2015;4.

16. Bhattacharjee R, Kheirandish-Gozal L, Spruyt K, et al. Adenotonsillectomy outcomes in treatment of obstructive sleep apnea in children: a multicenter retrospective study. Am J Respir Crit Care Med 2010;182:676-83.

17. Shapiro NL, Bhattacharyya N. Cold dissection versus coblationassisted adenotonsillectomy in children. Laryngoscope 2007;117:406-10.

18. Park A, Proctor MD, Alder S, et al. Subtotal bipolar tonsillectomy does not decrease postoperative pain compared to total monopolar tonsillectomy. Int J Pediatr Otorhinolaryngol 2007;71:1205-10.

19. Glade RS, Pearson SE, Zalzal GH, et al. Coblation adenotonsillectomy: an improvement over electrocautery technique? Otolaryngol Head Neck Surg 2006;134:852-5.

20. Martin G. Complications associées aux instruments chirurgicaux utilisés pour les amygdalectomies. Une, 2012.

21. Guyatt GH, Oxman AD, Kunz R, et al. Grade guidelines: 2. framing the question and deciding on important outcomes. J Clin Epidemiol 2011;64:395-400.

22. Higgins J, Green S. Cochrane Handbook for systematic reviews of interventions, version 5.1.0. The Cochrane Collaboration, 2011.

23. Schunemann HJ. Grade guidelines: 18. How ROBINS-I and other tools to assess risk of bias in nonrandomized studies should be used to rate the certainty of a body of evidence. $J$ Clin Epidemiol 2018.

24. Sterne JAC, Hernán MA, Reeves BC, et al. ROBINS-I: a tool for assessing risk of bias in non-randomised studies of interventions. BMJ 2016;355

25. Guyatt GH, Oxman AD, Vist GE, et al. Grade: an emerging consensus on rating quality of evidence and strength of recommendations. $B M J$ 2008;336:924-6.

26. DerSimonian R, Laird N. Meta-Analysis in clinical trials. Control Clin Trials 1986;7:177-88

27. Thorlund K, Walter SD, Johnston BC, et al. Pooling health-related quality of life outcomes in meta-analysis-a tutorial and review of methods for enhancing Interpretability. Res Synth Methods 2011;2:188-203.

28. Thompson SG, Higgins JPT. How should meta-regression analyses be undertaken and interpreted? Stat Med 2002;21:1559-73.

29. Traeger N, Schultz B, Pollock AN, et al. Polysomnographic values in children 2-9 years old: additional data and review of the literature. Pediatr Pulmonol 2005;40:22-30.

30. Guyatt G. Users' guides to the medical literature. 2nd edn, 2008.

31. Balshem H, Helfand M, Schünemann HJ, et al. Grade guidelines: 3 . rating the quality of evidence. J Clin Epidemiol 2011;64:401-6.

32. Guyatt GH, Oxman AD, Vist G, et al. GRADE guidelines: 4. Rating the quality of evidence--study limitations (risk of bias). J Clin Epidemiol 2011;64:407-15.

33. Guyatt GH, Oxman AD, Kunz R, et al. GRADE guidelines 6. Rating the quality of evidence--imprecision. $J$ Clin Epidemiol 2011;64:1283-93.

34. Guyatt GH, Oxman AD, Kunz R, et al. GRADE guidelines: 7. Rating the quality of evidence--inconsistency. J Clin Epidemiol 2011;64:1294-302. 
35. Higgins JPT, Thompson SG, Deeks JJ, et al. Measuring inconsistency in meta-analyses. $B M J$

2003;327:557-60.

36. Guyatt GH, Oxman AD, Kunz R, et al. GRADE guidelines: 8. Rating the quality of evidence--indirectness. $J$ Clin Epidemiol 2011;64:1303-10.
37. Guyatt GH, Oxman AD, Montori V, et al. GRADE guidelines: 5 . Rating the quality of evidence--publication bias. J Clin Epidemiol 2011;64:1277-82.

38. Guyatt G, Oxman AD, Sultan S, et al. Grade guidelines: 11. making an overall rating of confidence in effect estimates for a single outcome and for all outcomes. J Clin Epidemiol 2013;66:151-7. 